\title{
Vertically Aligned Pd Nanowires for Glucose Oxidase Bioanode
}

Gymama Slaughter* and Kweku Amoah

Department of Computer Science and Electrical Engineering, University of Maryland Baltimore County and Bioelectronics Laboratory, USA

\begin{abstract}
A bioanode for glucose based biofuel cell have been fabricated by combining glucose oxidase and highly ordered Palladium $(\mathrm{Pd})$ nanowire array electrode. The $\mathrm{Pd}$ nanowires were $5.57 \mu \mathrm{m}$ in length and $64.28 \mathrm{~nm}$ in diameter. The hydrogel composite modified Pd nanowire array bioanodes were characterized with cyclic voltammetry in the presence of different scan rates and different substrate concentrations under physiological conditions. The electrochemical measurements showed a linear amperometric response in the range of $1 \mathrm{mM}$ to $13 \mathrm{mM}$. In addition, surface and structural characterization were performed by scanning electron microscopy leading to the conclusion that the Pd nanowires are highly ordered and vertically aligned and thereby promotes the number of electroactive sites for the catalysis of the oxidation of glucose. The combination of glucose oxidase and Pd nanowire arrays revealed the capability of these enzymatic bioanodes to perform direct electron transfer to Pd nanostructure surfaces and be used in a glucose biofuel cell.
\end{abstract}

Keywords: Glucose oxidase; Pd nanowires; Bioanode; Cyclic voltammetry

\section{Introduction}

Enzymatic biofuel cells, especially glucose- $\mathrm{O}_{2}$ biofuel cells are fabricated from a variety of electrode materials [1]. Currently, there is a strong focus on the development of these biofuel cells for use as implantable and micro/ nano device power sources [2-9]. The desire to develop energy sources based on glucose biofuel cells for implantable devices can be attributed to the fact that the bioanode enzymes such as glucose oxidase and glucose dehydrogenase can oxidize glucose at physiological conditions. This permits the extracted glucose from the bloodstream or the interstitial fluid to be used as fuel substrate for energy harvesting under a physiologic conditions.

Typically, glucose-based biofuel cells consist of a bioanode and a biocathode at which oxidation-reduction (redox) reactions occur in the presence of the fuel source. The biofuel cell circuit is completed with an external load to allow the conduction of electrons from the bioanode to the biocathode (Figure 1). Therefore, the choice of the anodic and cathodic material depends on several factors including but not limited to the biocatalyst to catalyze the electrode reactions; the integration of the biocatalyst with the appropriate physicochemical transduction element for harvesting energy from the various concentration of glucose

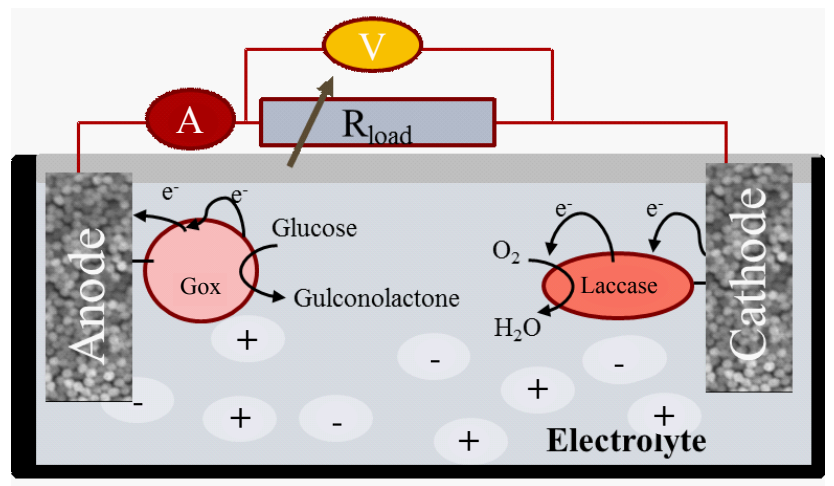

Figure 1: Schematic representation of the biofuel cell structure. Glucose is oxidized at the anode to gluconolactone and the electrons generated are transferred glucose oxidase to the $\mathrm{Pd}$ nanowires. The electrons are then transferred from the $\mathrm{Pd}$ nanowires to laccase at the cathode where oxygen is reduced to form water. in blood or interstitial fluid; effective transduction element surface area in order to increase the number of binding sites for the biocatalyst; ease of fabrication; and enhanced durability to ensure extended functional lifetimes of biofuel cell devices for implantation [9-14]. Furthermore, a biocompatible encapsulating layer for the biofuel cell is required in order to enhance the biocompatibility and durability of the entire device in vivo.

In this work, we describe the fabrication of a glucose biofuel cell bioanode based on the combination of glucose oxidase and Palladium (Pd) nanowire array electrodes, which has not been reported with respect to glucose-based biofuel cell applications. Pd nanowire array electrodes are high surface area three-dimensional bioanode, which are fabricated from Anodized Aluminum Oxide (AAO) template electrodeposition method [15-17]. These fabricated AAO templates are inherently ordered in nature and exhibits a highly self-arranged hexagonal array that permit them to be used as a template for the electrodeposition of metal species [18]. The nanowires formed on the electrode surface were characterized by Scanning Electron Microscopy (SEM). Glucose oxidase was covalent immobilized using a hydrogel composite membrane onto the Pd nanowire arrays. Cyclic voltammetry measurements were performed at different scan rates and in different glucose concentration to evaluate the electrocatalytic activity of the hydrogel composite modified Pd nanowire arrays [2,19]. In principle, the combination of glucose oxidase and Pd nanowire arrays leads to the development of a glucose oxidase bioanode that can oxide glucose, enhance the bioanode conductivity and facilitate the direct electron transfer of the biocatalyst without the use of a mediator [8].

*Corresponding author: Gymama Slaughter, Department of Computer Science and Electrical Engineering, of Maryland Baltimore County and Bioelectronics Laboratory, 1000 Hilltop Circle, Baltimore, MD 21250, USA, Tel: +1 4104558483 ; Fax: +1 410455 396; E-mail: gslaughter@vsu.edu

Received August 10, 2012; Accepted September 04, 2012; Published September 05, 2012

Citation: Slaughter G, Amoah K (2012) Vertically Aligned Pd Nanowires for Glucose Oxidase Bioanode. J Biosens Bioelectron 3:124. doi:10.4172/2155 6210.1000124

Copyright: (c) 2012 Slaughter G, et al. This is an open-access article distributed under the terms of the Creative Commons Attribution License, which permits unrestricted use, distribution, and reproduction in any medium, provided the original author and source are credited. 


\section{Experimental}

\section{Materials and methods}

AAO templates $(10 \mathrm{~mm} \times 10 \mathrm{~mm})$ with average pore diameter of 80 $\mathrm{nm}$, pore density $2 \times 10^{9} \mathrm{~cm}^{-2}$ and estimated porosity $15 \%$, were purchased from Synkera Technologies, Inc. Glucose oxidase (GOx, EC 1.1.3.4 from Aspergillus niger), d-(+)-glucose, Hydroxyethylmethacrylate (HEMA), Tetraethyleneglycol Diacrylate (TEGDA), 2,2-Dimethoxy-2Phenylacetophenone (DMPA, 99\%), (3-aminopropyl)trimethoxysilane ( $\gamma$-APS, 97\%) and Poly(Ethylene Glycol) Methacrylate (PEGMA) were purchased from Sigma-Aldrich. The diacyrlate and methacrylate reagents were passed through an inhibitor removal column in order to remove the hydroquinone and monomethyl ether hydroquinone polymerization inhibitors before use. Glucose solutions were prepared using $0.1 \mathrm{M}$ phosphate buffer saline (PBS, $\mathrm{pH}$ 7.3). Acryloyl(polyethyleneglycol)-N-hydroxysuccinamide (MW 3500), was purchased from Jenkem Technology. All supplementary chemicals were of analytical grades and solutions were prepared with $18.2 \mathrm{M} \Omega$ $\mathrm{cm}$ Milli-Q water.

\section{Electrodeposition of Pd nanowires}

The AAO templates were $98 \pm 1 \mu \mathrm{m}$ in depth with a pore diameter of $80 \pm 8 \mathrm{~nm}$. Pd was electrochemically deposited from an aqueous solution containing $1 \mathrm{~g} \mathrm{~L}^{-1} \mathrm{Pd}\left(\mathrm{NH}_{3}\right)_{4} \mathrm{Cl}_{2}$ (99.99\%) and $10 \mathrm{~g} \mathrm{~L}^{-1} \mathrm{NH}_{4} \mathrm{Cl}$ (99.99\%). Ammonium hydroxide was used to adjust the $\mathrm{pH}$ to 8 . Prior to electrodeposition, the AAO template was pretreated in acetone to clean the surface of the substrate followed by drying under nitrogen flow. A thin layer of $\mathrm{Au}(400 \AA)$ was sputtered onto one side of the AAO template to provide an electrical conduction path for the electrodeposition of the Pd nanowires by exposing the pores of the AAO template to the underlying Au film. This provides a means for the electrodeposition of metal species through the pores. To create an electrical connection to the AAO working electrode, a polyethylene terephthalate film $(0.8 \mathrm{~cm} \times 10 \mathrm{~cm})$ was prepared with a thin film of $\mathrm{Au}$ ( $400 \AA$ ) and was attached to the Au sputtered side of the AAO substrate to allow for easy handling of the substrate material. A polyimide tape was used for electrode passivation and to define the electroactive area of the working electrode.

The prepared AAO substrate served as the working electrode in a three-electrode setup with a platinum mesh and a $\mathrm{Ag} / \mathrm{AgCl}_{\text {(sat) }}$ electrode as the counter and reference electrodes, respectively in an electrochemical cell with water jacket. The electrodeposition was performed at an applied potential of $-600 \mathrm{mV}$ with a BASi Epsilon Electrochemical Workstation and under a constant temperature of $30^{\circ} \mathrm{C}$ (Fischer Scientific Isotemp 3016D) with constant stirring for 8 hours. The mechanism of Pd nanowire deposition is described in the work of Tasaltin et al. [20] and should be Cheng et al. [21] and Peppas et al. [22]. After electrodeposition, the substrate was rinsed in deionized water and the AAO template was etched away in $2 \mathrm{M} \mathrm{NaOH}$ for 30 minutes at room temperature with agitation resulting in free standing $\mathrm{Pd}$ nanowire array electrodes. These Pd nanowire array electrodes were then rinsed three times with ethanol followed by deionized water.

\section{Preparation of glucose oxidase bioanode}

The surface of the Pd nanowire array electrodes was subsequently functionalized by treatment with $10 \mathrm{mM}$ 3-(aminopropyl) trimethoxysilane ( $\gamma$-APS) at $40^{\circ} \mathrm{C}$ for 30 minutes in order to introduce silane surface functionalities. After silanization, the Pd nanowire array electrodes were rinsed by sequential washing for 1 minute in ethanol, then in ethanol/water mixture $(1: 1, v / v)$. Finally, the Pd nanowire array electrodes were cured at $110^{\circ} \mathrm{C}$ for 20 minutes in a convection oven. This was followed by a derivatization step to create a continuous path of covalent bonding between the Pd nanowire electrode surfaces and the hydrogel composite membrane. The Pd nanowire array electrodes were incubated in the dark for 2 hours in $50 \mathrm{mM}$ HEPES ( $\mathrm{pH}=7.4$ ) and $1 \mathrm{mM}$ Acryloyl(polyethyleneglycol)-N-hydroxysuccinamide solution at room temperature. The derivatized Pd nanowire array electrodes were subsequently rinsed with $50 \mathrm{mM}$ HEPES buffer.

Glucose oxidase enzyme solution with a concentration of 15 $\mathrm{mg} / \mathrm{mL}$ was entrapped within a composite UV cross-linked HEMAbased hydrogel membrane material and subsequent deposition onto the Pd nanowire array electrodes. The monomer mixture comprised of HEMA, PEGMA, TEGDA in a mole ratio of 80:10:10. Glucose oxidase was dissolved in ethylene glycol: water $(1: 1 \mathrm{v} / \mathrm{v})$ and then introduced into the monomer mixture. Photoinitiator DMPA (4 $\mathrm{wt} \%$ ) was then completely dissolved in the mixture and $3 \mu \mathrm{L}$ of the enzyme-monomer mixture was applied to the Pd nanowire array electrodes. UV irradiation $\left(2.3 \mathrm{~W} / \mathrm{cm}^{2}, 366 \mathrm{~nm}\right.$, UVP Model CX-2000) was performed for 20 minutes to polymerize the methacrylates. The PEGMA monomer was added to the hydrogel formulation to provide for long-term stabilization of membrane-immobilized glucose oxidase. This establishes PEG chains, set pendant to the polymer network, in the form of network supported polymer brushes. PEG chains are well known to stabilize proteins, preventing their denaturation and promoting long-term bioactivity [23].

The cyclic voltammetry experiments were performed on a BASi Epsilon at room temperature using a three-electrode cell. SEM measurements were performed with Hitachi SU70 to characterize the surface structure of the deposited Pd nanowires.

\section{Results and Discussion}

The physical appearance and surface characteristics of the Pd nanowire array electrodes were studied with an SEM. Figure 2 illustrates the typical SEM image of the free standing Pd nanowire arrays. As can be seen, highly ordered Pd nanowires were electrodeposited into the pores of the AAO template, upon completely etching away the AAO template. The average diameter of the nanowires is about 64.28 $\mathrm{nm}$ with a symmetric distribution. These nanowires are rod-like, uniform and vertically aligned on the electrode surface. The average length of the nanowires is $5.57 \mu \mathrm{m}$. The length of these Pd nanowires

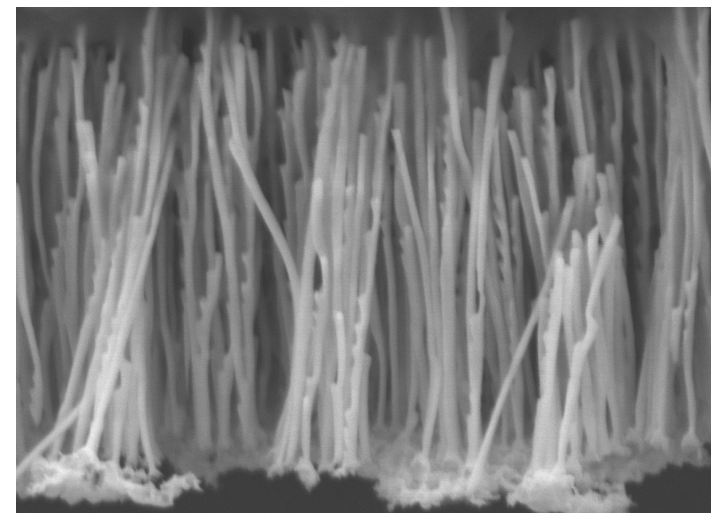

Figure 2: SEM micrograph (cross-section) of electrodeposited $\mathrm{Pd}$ nanowires from ammoniacal solution at a potential of $-600 \mathrm{mV} v \mathrm{vs} . \mathrm{Ag} / \mathrm{AgCl}$ and $\mathrm{t}=8 \mathrm{~h}$ using AAO template. 


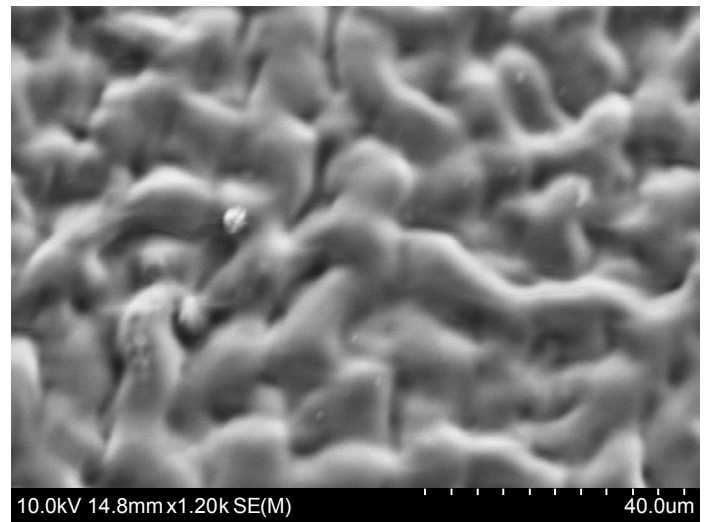

Figure 3: SEM micrograph of the morphology of the bioanode.

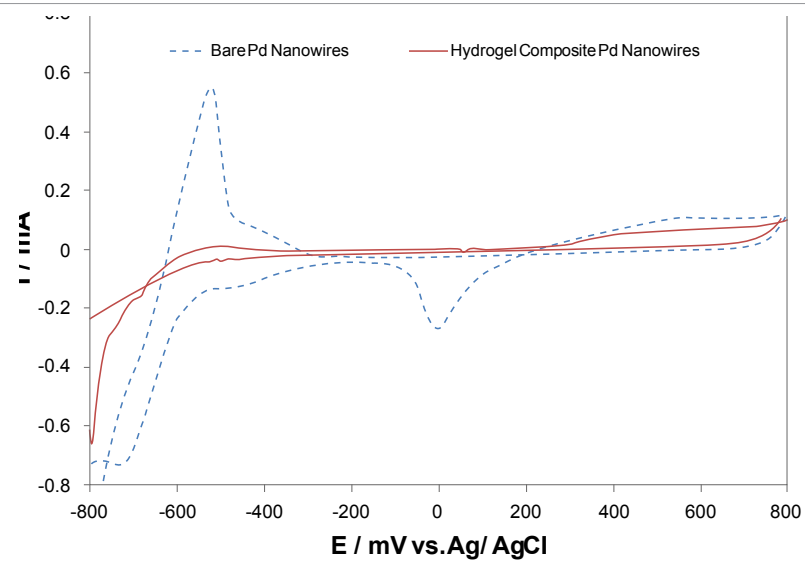

Figure 4: CVs of $100 \mathrm{mM}$ phosphate buffer $(\mathrm{pH}=7.4)$ at $(---)$ bare $\mathrm{Pd}$ nanowires and (__ ) hydrogel composite modified $\mathrm{Pd}$ nanowire array electrodes. Scan rate: $2 \mathrm{mV} \mathrm{s}^{-1}$.

is much longer than those previously reported $[15,19]$ because longer deposition time was necessary due to the depth of the AAO template. The Pd nanowire length is an important factor for the performance of the bioanode since it provides a larger electroactive surface area, thus resulting in higher catalytic capability of the glucose oxidase bioanode. The resulting hydrogel composite membrane on the Pd nanowire arrays (Figure 3 ) displays a marked difference in surface characteristics. This indicates clearly that the hydrogel composite membrane can be effectively integrated with the Pd nanowire array electrode surfaces through surface functionalization and crosslinking. The underlying Pd nanowire array electrode maintains high surface area after coating with hydrogel composite membrane.

The characteristic Cyclic Voltammograms (CVs) of the bare $\mathrm{Pd}$ nanowire array electrodes and hydrogel composite modified Pd nanowire array electrodes responses were measured in the presence of $100 \mathrm{mM}$ phosphate buffer $(\mathrm{pH}=7.4)$ at the scan rate of $2 \mathrm{mV} \mathrm{s}^{-1}$. Figure 4 shows the corresponding CV curves for the bare Pd nanowires and the hydrogel composite modified Pd nanowire array electrodes. There is a large observable faradaic current on the Pd nanowire array electrodes with an oxidation peak at $+0.05 \mathrm{~V}$ along with a reduction peak at $-0.55 \mathrm{~V}$, which attributes to the reduction of palladium surface oxides. The CV of the hydrogel composite modified Pd nanowire array electrodes shows that a pair of oxidation and reduction peaks at +0.05
$\mathrm{V}$ and $-0.55 \mathrm{~V}$, respectively were obtained. This provides confirmation that the hydrogel composite membrane restricts the flow of dissolved $\mathrm{O}_{2}$ to the $\mathrm{Pd}$ nanowire arrays surfaces, thereby resulting in small electrocatalytic activity for Pd surface oxide.

The CVs for hydrogel composite modified Pd nanowire array electrodes responses were measured in the presence of $5 \mathrm{mM}$ glucose substrate $(\mathrm{pH}=7.4)$ at different scan rates (Figure 5). With increasing scan rates, the peak-to-peak separation and the observed linear relationship between the peak currents $\left(\mathrm{I}_{\mathrm{pa}}\right.$ and $\left.\mathrm{I}_{\mathrm{pc}}\right)$ and the scan rate $(\mathrm{v})$ from 2 to $800 \mathrm{mV} \mathrm{s}^{-1}$ in the insert of (Figure 3) illustrate that the redox process of glucose oxidase in this hydrogel composite membrane is a reversible process and is in agreement with the typical surface-confined process.

The resulting hydrogel composite modified Pd nanowire array electrodes exhibited good electrocatalysis toward the oxidation of glucose. The CVs for the hydrogel composite modified Pd nanowire array electrodes responses were measured in the absence of glucose substrate and at glucose concentrations of $1,3,5$ and $13 \mathrm{mM}$ under

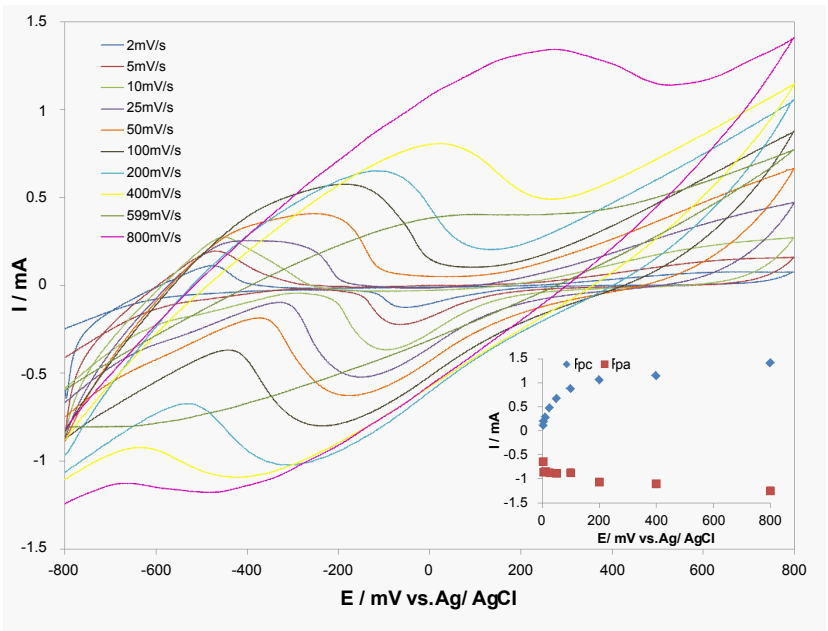

Figure 5: CVs of hydrogel composite modified Pd nanowire array bioanode in $5 \mathrm{mM}$ glucose $(\mathrm{pH}=7.4)$ at various scan rates. The scan rate is $2,5,10,25$, $50,100,200,400,599,800 \mathrm{mV} \mathrm{s}^{-1}$ (from inner to outer). Insert: Plot of peak currents vs. scan rate.

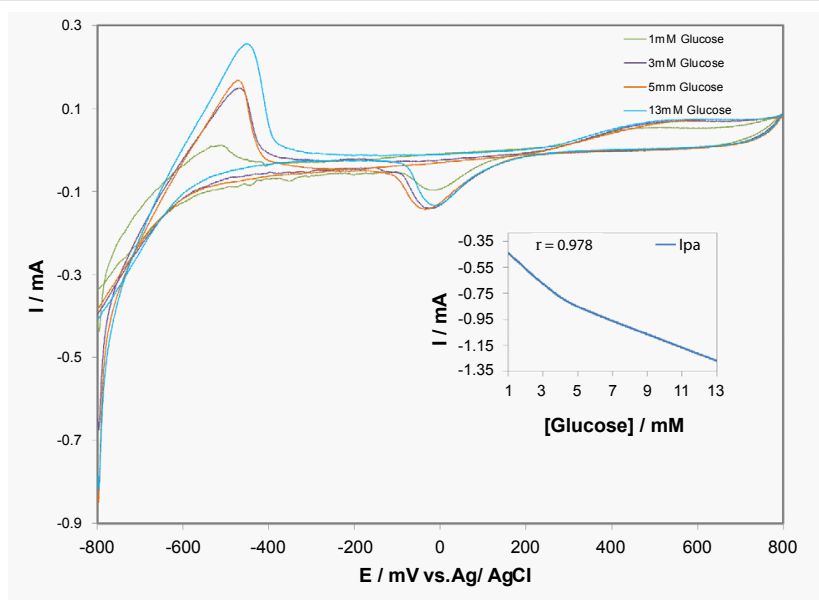

Figure 6: CVs at the hydrogel composite modified $\mathrm{Pd}$ nanowire array bioanode in various concentrations of glucose: $1,3,5$ and $13 \mathrm{mM}$ from inner to outer. Insert: Calibration curve $(R=0.978)$. Scan rate of $2 \mathrm{mV} \mathrm{s}^{-1}$. 
physiological conditions at the scan rate of $2 \mathrm{mV} \mathrm{s}^{-1}$ (Figure 6). The oxidation current became larger and larger with increased concentration of glucose as a result of the oxidation of glucose to gluconic acid. The amperometric response reached saturation in the presence of glucose concentration greater than $13 \mathrm{mM}$. The calibration curve corresponding to the amperometric response (Figure 6) has a linear dynamic range from $1 \mathrm{mM}$ to $13 \mathrm{mM}(\mathrm{r}=0.978)$. The current density and power density for glucose oxidation at $5 \mathrm{mM}$ glucose concentration, which is in the normal range of glucose concentration in the bloodstream is $851 \mu \mathrm{A} \mathrm{cm}-2$ and $1.2 \mathrm{~mW} \mathrm{~cm}$, respectively. This demonstrates that the Pd nanowire array electrodes promote the number of electroactive sites for the oxidation of glucose. These results further demonstrate that the glucose oxidase Pd nanowire array bioanode exhibits higher electrocatalytic activities at physiologic conditions, which directly improves glucose biofuel cell efficiency.

\section{Conclusion}

The major driving forces for the development of glucose based biofuel cells is energy sources for biomedical implantable devices. $5.57 \mu \mathrm{m}$ long palladium nanowires with an average diameter of $64.28 \mathrm{~nm}$ were successfully electrodeposited using AAO template electrodeposition method. The hydrogel composite modified Pd nanowire arrays exhibited good electroactivity toward the oxidation of glucose. The hydrogel composite membranes achieved the direct electron transfer of redox enzyme, thereby not only demonstrating its potential to be used as a bioanode in glucose based biofuel cells, but also can be extended to biosensors.

\section{Acknowledgment}

The authors will like to thank Tim Buckheit, UMBC Microfabrication Cleanroom Manager and Dr. Wen-An Chiou at the Nanoscale Imaging, Spectroscopy and Properties Laboratory at the University of Maryland, College Park.

\section{References}

1. Bullen RA, Arnot TC, Lakeman JB, Walsh FC (2006) Biofuel cells and their development. Biosens Bioelectron 21: 2015-2045.

2. Moehlenbrock MJ, Arechederra RL, Sjöholm KH, Minteer SD (2009) Analytical techniques for characterizing enzymatic biofuel cells. Anal Chem 81: 95389545.

3. Kannan AM, Renugopalakrishnan V, Filipek S, Li P, Audette GF, et al. (2009) Bio-batteries and bio-fuel cells: leveraging on electronic charge transfer proteins. J Nanosci Nanotechnol 9: 1665-1678.

4. Davis F, Higson SP (2007) Biofuel cells--recent advances and applications. Biosens Bioelectron 22: 1224-1235.

5. Atanassov P, Apblett C, Banta S, Brozik S, Calabrese Barton S, et al. (2007) Electrochemical Society Interface Summer 16: 28-31.

6. Justin GA (2004) Biofuel Cells as a Possible Power Source for Implantable Electronic Devices. Master's Thesis, University of Pittsburgh.

7. Ribeiro A, Ferreira R, Pires P (2010) Biofuel Cells In Implantable Medical Devices. Instituto Superio Tecnico.

8. Barton SC, Gallaway J, Atanassov P (2004) Enzymatic biofuel cells for implantable and microscale devices. Chem Rev 104: 4867-4886.

9. Logan BE, Hamelers B, Rozendal R, Schröder U, Keller J, et al. (2006) Microbial fuel cells: methodology and technology. Environ Sci Technol 40: 5181-5192.

10. Cinquin P, Gondran C, Giroud F, Mazabrard S, Pellissier A, et al. (2010) A Glucose BioFuel Cell Implanted in Rats. PLoS ONE 5: e10476.

11. Rasmussen M, Ritzmann RE, Lee I, Pollack AJ, Scherson D (2012) An implantable biofuel cell for a live insect. J Am Chem Soc 134: 1458-1460.

12. Halámková L, Halámek J, Bocharova V, Szczupak A, Alfonta L, et al. (2012) Implanted biofuel cell operating in a living snail. J Am Chem Soc 134: 50405043.
13. Liu Y, Harnisch F, Fricke K, Schröder U, Climent V, et al. (2010) The study of electrochemically active microbial biofilms on different carbon-based anode materials in microbial fuel cells. Biosens Bioelectron 25: 2167-2171.

14. Schröder U, Niessen J, Scholz F (2003) A generation of microbial fuel cells with current outputs boosted by more than one order of magnitude. Angew Chem Int Ed Engl 42: 2880-2883.

15. Sun Z, Cheng F, Dai X (2009) Highly ordered Pd nanowire array by template fabrication for propanol electrooxidation. J Autom Methods Manag Chem 2009: 496281.

16. Kim J, Jia H, Wang $P$ (2006) Challenges in biocatalysis for enzyme-based biofuel cells. Biotechnol Adv 24: 296-308.

17. Cherevko S, Fu J, Kulyk N, Cho SM, Haam S, et al. (2009) Electrodeposition mechanism of palladium nanotube and nanowire arrays. J Nanosci Nanotechno 9: 3154-3159.

18. Rabin O, Herz PR, Lin YM, Akinwande Al, Cronin SB, et al. (2003) Formation of Thick Porous Anodic Alumina Films and Nanowire Arrays on Silicon Wafers and Glass. Adv Funct Mater 13: 631-638.

19. Li Y, Chen S, Sarawathi R (2011) Membraneless enzymatic biofuel cells based on multi-walled carbon nanotubes. Int J Electrochem Sci 6: 3776-3788.

20. Taşaltın N, Öztürk S, Kılınç N, Yüzer H, Öztürk Z (2010) Fabrication of vertically aligned $\mathrm{Pd}$ nanowire array in AAO template by electrodeposition using neutra electrolyte. Nanoscale Res Lett 5: 1137-1143.

21. Cui C, Li H, Yu S (2010) A general approach to electrochemical deposition of high quality free-standing noble metal $(\mathrm{Pd}, \mathrm{Pt}, \mathrm{Au}, \mathrm{Ag})$ sub-micron tubes composed of nanoparticles in polar aprotic solvent. Chem Commun 46: 940 942.

22. Cheng F, Wang H, Sun Z, Ning M, Cai Z, et al. (2008) Electrodeposited fabrication of highly ordered $\mathrm{Pd}$ nanowire arrays for alcohol electrooxidation. Electrochem Commun 10: 798-801

23. Peppas NA, Keys KB, Torres-Lugo M, Lowman AM (1999) Poly(ethylene glycol)-containing hydrogels in drug delivery. J Control Release 62: 81-87. 